\title{
MECHANISM OF OXIDATIVE RING CONTRACTION OF MONOCYCLIC POLYOXOFNEDIOLS BY ACTIVE MANGANESE DIOXIDE
}

C. NALLAIAH

Department of Chemistry, Imo State University, Etiti, Imo State, Nigeria.

(Date of receipt : 28.11.84)

(Date of acceptance : 10.11.86)

\begin{abstract}
A new mechanism which involves a benzylic acid type of rearrangement of fully dehydrated triquinoyl is presented for the oxidative ring contraction reactions of monocyclic polyoxoenediols. Manganese dioxjde is thought to merely act as an oxidising agent, oxidising these polyoxoenediols to triquinoyl and is not involved in the actual ring contraction reaction.
\end{abstract}

\section{Introduction}

Oxidative ring contraction reactions of benzenehexol (1) and other monocyclic polyoxoenediols like rhodizonic acid (2), tetrahydroxy 1,4-benzoquinone (3) etc, with active manganese dioxide under basic conditions to form croconic acid (6) are well documented. ${ }^{1,2,3}$. Fatiadi et al ${ }^{3,4}$ have proposed a mechanism for oxidative ring contraction of benzenehexol with active manganese dioxide, involving ionic and free radical pathways. This mechanism is ambiguous in that certain steps in the mechanism are not clearly defined. Even though it is possible to generate a carbonium ion adjacent to a carbonyl group, the intermediacy of a bivalent carbonium ion is rather unusual. Furthermore according to their mechanism manganese dioxide is directly involved in the actual ring contraction reaction.

\section{Results and Discussion}

It is significant that compounds (1), (2) and (3), all of which form croconic acid in good yields can also be oxidised to triquinoyl (4) (Scheme-1).

The structure of triquinoyl has been controversial for many years and the absence of a characteristic absorption band in the carbonyl region of the infra red spectrum of this compound, has led to the proposal ${ }^{7}$ that it has a 
<smiles>O=C1C(O)=C(O)C(=O)C(O)=C1O</smiles>

(I)
(3)<smiles>O=C1C(=O)C(=O)C(=O)C(=O)C1=O</smiles><smiles>O=c1c(O)c(O)c(=O)c(=O)c1=O</smiles>

(2)<smiles>OC1(O)C(O)(O)C(O)(O)C(O)(O)C(O)(O)C1(O)O</smiles>

(5)

fully hydroxylated gemdicl structure (5). The ${ }^{13} \mathrm{C}-\mathrm{NMR}$ Spectrum of triquinoyl in DMSO did not give a single peak for the six equivalent $\mathrm{C}(\mathrm{OH})_{2}$ (or six equivalent $\mathrm{C}=\mathrm{O}$ if present in the fully dehydrated form) but instead gave three peaks which were assigned to rhodizonic acid dihydrate. ${ }^{8}$ The mass spectrum of triquinoyl octahydrate did not show a parent ion peak of either (5) $(\mathrm{m} / \mathrm{e} 312)$ or $(4)(\mathrm{m} / \mathrm{e} 168)$. Instead the mass spectrum revealed intense peaks at $m / e 172$ and $m / e 170$ due to $(3)$ and $(2)$ respectively! Thus it is apparent that triquinoyl exists in the fully hydroxylated state (5) in the solid form and in solution it is in equilibrium with various species such as (2), (3), etc. 
It has been reported ${ }^{4}$ that triquinoyl can be oxidised to croconic acid by reacting it with active manganese dioxide under basic conditions. However, we have synthesised croconic acid in substantial yields ( $\sim 50-60 \%)$ by refluxing triquinoyl in $50 \%$ aqueous solution of potassium hydroxide, for $(30-45)$ minutes in the absence of active manganese dioxide. This indicates that the formation of croconic acid from (1), (2) and (3) could be via triquinoyl, active manganese dioxide merely acting as an oxidising agent, oxidising these compounds i.e.(1), (2) and (3) to triquinoyl. The mechanism that we propose for these transformations is depicted in Scheme -2 .

SCHEME - 2

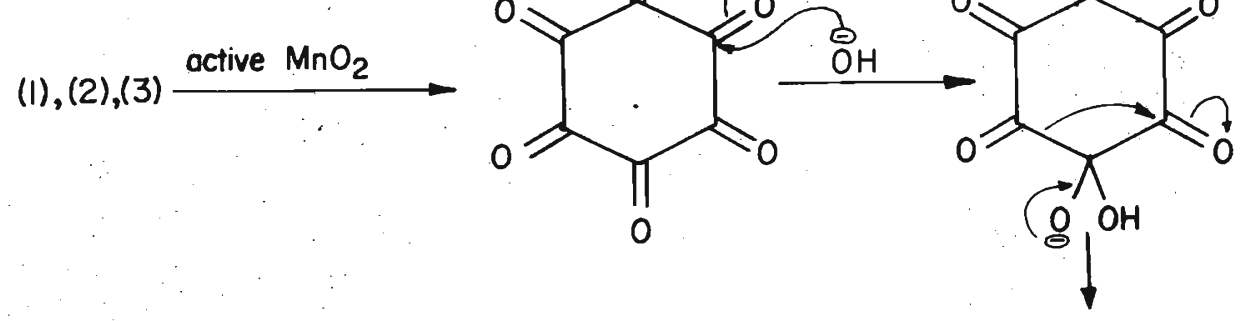

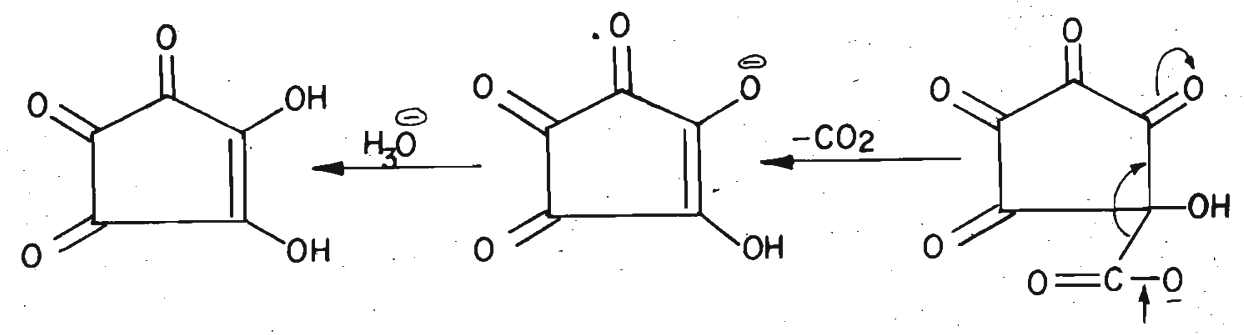


Active manganese dioxide oxidises benzenehexol and other related compounds like (2) and (3) to (4), which under basic conditions undergoes a benzilic type of rearrangement to form the intermediate (7). This intermediate is a $\beta$-keto acid and undergoes ready decarboxylation to form croconic acid.

It is possible that in the absence of active manganese dioxide, triquinoyl (in form (5) ), reverts to the fully dehydrated form (4) due to aerial oxidation. However when the reactions were carried out with triquinoyl and aqueous base in an inert atmosphere of nitrogen, the major product was again found to be croconic acid. Thus it appears that the fully hydroxylated form of triquinoyl (5) changes to the dehydrated form (4) on refluxing. In the oxidative ring contraction reactions of (1), (2) and (3) with active manganese dioxide, samples of the reaction mixture were withdrawn at intervals of 5 minutes. Analysis of these samples by tlc using silica gel and various mixtures of petroleum ether $\left(40^{\circ} \mathrm{C}-60^{\circ} \mathrm{C}\right) /$ ethyl acetate $(10: 1$, $8: 1,5: 1)$ with an authentic sample of triquinoyl as reference did not reveal the presence of triquinoyl. This is hardly surprising since such experiments with triquinoyl and aqueous base in the absence of active manganese dioxide, the presence of triquinoyl could not be detected even after 5 minutes of starting the reaction.

Thus the formation of croconic acid by reacting triquinoyl with aqueous base and the rapid reaction of triquinoyl with aqueous base indicates that in the oxidative ring contraction reactions of (1), (2) and (3) with active manganese dioxide under basic conditions, the oxidant merely oxidises these compounds to triquinoyl and is not directly involved in the ring contraction reaction.

\section{Experimental}

Most of the organic compounds used were of analar grade. Reagent grade chemicals were purified by redistillation or recrystallisation as appropriate. IR measurements were recorded on a Perkin-Elmer 457 grating spectrophotometer using $\mathrm{KB}$ discs. Analytical tlc was carried out with silica gel $\mathrm{F}_{254}(0.25 \mathrm{~mm})$ deposited on plastic sheets (commercially available), and developed using various mixtures of petroleum ether $\left(40-60^{\circ} \mathrm{C}\right)$ and ethylacetate.

Triquinoyl ${ }^{1}$ and active manganese dioxide ${ }^{2}$ were prepared according to the methods given in literature. 


\section{Reaction of Triquinoyl with Base}

Triquinoyl (1.5g) was mixed with water $\left(50 \mathrm{~cm}^{3}\right)$, ethyl alcohol $\left(25 \mathrm{~cm}^{3}\right)$ and $50 \%$ aqueous potassium hydroxide $\left(15 \mathrm{~cm}^{3}\right)$. The mixture was refluxed for 45 minutes and filtered immediately. The filtrate was concentrated and on cooling in an ice bath, yielded bright yellow crystals of potassium croconate. A further crop of potassium croconate was obtained by concentrating the filtrate $0.789 \mathrm{~g}(52 \%)$, m.pt $360^{\circ} \mathrm{C}$, IR $(\mathrm{KBr})$ identical to that of an authentic sample of potassium croconate.

\section{Acknowledgements}

The author would like to thank Professor. L. Crombie for generating interest in cyclic polyoxoenediols.

\section{References}

1. EISTERT, B., BOCK, G. (1958) Angew. Cbem., 70(19) : 595.

2. FATIADI, A.J., (1971) J. Chem. $\operatorname{Soc}(\mathbf{B}): 889$.

3. FATIADI, A.J., (1976) Synthesis, 3: 133.

4. FATIAdi, A. J., ISBELL, H.S. \& SAGER, W. F. (1963) J. Res. Nat. Bur. Stand., 67A : 153.

5. HOLZEL, R., LEFTWICK, A. P., WEEDON, B. C. L. (1969) J. Chem. Soc. Chem. Comm., 128.

6. KUBOTA, T. \& TAKEDA, K. (1960) Tetrabedron; $10: 1$.

7. PERSON, W. B. \& Williams, D. G. (1957) J. Pby. Cbem., $61: 1017$.

8. STADEli, W., hollenstein, R. \& Von PhilipSBORN, W. (1971) Hel. Chem. Acta., 60(3) : 948. 